\title{
Analysis of Record Data from the Scaled Logistic Distribution
}

\author{
A. Asgharzadeh ${ }^{\dagger, *}$, M. Abdi ${ }^{\ddagger}$ and R. Valiollahi ${ }^{\star}$ \\ $\dagger$ University of Mazandaran \\ ‡ Higher Education Complex of Bam \\ * Semnan University
}

\begin{abstract}
In this paper, we consider the estimation of the unknown parameter of the scaled logistic distribution on the basis of record values. The maximum likelihood method does not provide an explicit estimator for the scale parameter. In this article, we present a simple method of deriving an explicit estimator by approximating the likelihood function. Bayes estimator is obtained using importance sampling. Asymptotic confidence intervals, bootstrap confidence interval and credible interval are also proposed. Monte Carlo simulations are performed to compare the different proposed methods. Analysis of one real data set is also given for illustrative purposes.
\end{abstract}

Keywords. Bayes estimation; maximum likelihood estimation; Monte Carlo simulation; record values; importance sampling.

MSC 2010: 62F10; 62F15; 62G30.

\section{Introduction}

Let $X_{1}, X_{2}, \ldots$ be a sequence of independent and identically distributed (iid) random variables with cumulative distribution function (cdf) $F(x ; \theta)$ and probability density function (pdf) $f(x ; \theta)$, where $\theta$ is an unknown parameter. An observation $X_{j}$ will be called an upper record value if its value exceeds that of all previous observations. Thus, $X_{j}$ is an upper record if $X_{j}>X_{i}$ for

* Corresponding author 
every $i<j$. An analogous definition can be given for lower record values. If $\{U(n), n \geqslant 1\}$ is defined by

$$
U(1)=1, \quad U(n)=\min \left\{j: j>U(n-1), X_{j}>X_{U(n-1)}\right\},
$$

for $n \geqslant 2$, then the sequence $\left\{X_{U(n)}, n \geqslant 1\right\}$ provides a sequence of upper record statistics. The sequence $\{U(n), n \geqslant 1\}$ represents the record times.

Record statistics are defined as a model for successive extremes in a sequence of iid random variables (see, for example Glick, 1978). These statistics are of interest and important in many real life problems involving weather, economics, sports data and life testing studies. The times achieved in athletic events are most easily accessed in terms of record values. In reliability and life testing experiments, many products fail under stress. Hence, in such experiments, measurements may be made sequentially and only the record values (lower or upper) are observed. The statistical study of record values started with Chandler (1952) and has now spread in different directions. For more details on record values and its applications, see Arnold et al. (1998) and Nevzorov (2001). See also Ahmadi and Balakrishnan (2010), Soliman and Al-Aboud (2008) and Lee et al. (2011).

In recent years, a lot of work has been on statistical inference based on record data, see for example, Ahmadi and Arghami (2001, 2003a, b); Carlin and Gelfand (1993); Balakrishnan et al. (1995); Feuerverger and Hall (1998); Gulati and Padgett (1995, 2003), Asgharzadeh and Fallah (2011) and MirMostafaee and Ahmadi (2011), and references therein.

The logistic distribution, as well as the logistic growth curve, have many applications in reliability and in the biological sciences. The logistic model has often been selected as a substitute for the normal because of the similarity of the two distributions. Although much work has been done on inferential procedures for logistic distribution based on complete and censored data, but not much work has been done for record data. In this article, we consider record values from a scaled logistic distribution.

The rest of the paper is organized as follows. In Section 2, we discuss the point estimation of the unknown parameter of the scaled logistic distribution. We obtain the maximum likelihood estimator (MLE) and the approximate maximum likelihood estimator (AMLE). We also consider the Bayesian estimator based on the assumption that the unknown parameter has an inverse gamma prior. It is observed that the Bayesian estimator cannot be obtained in closed form and we propose to use importance sampling technique to compute the Bayes estimator. In Section 3, we provide 
asymptotic confidence interval, bootstrap confidence interval and Bayesian confidence interval. Bayesian confidence interval is obtained using importance sampling technique. Finally, in Section 4, one numerical example and a Monte Carlo simulation study are given to illustrate the results.

\section{Point Estimation}

In this subsection, we discuss the point estimation of the scale parameter of the logistic distribution.

\subsection{Maximum Likelihood Estimation}

Let the failure time distribution be the scaled logistic distribution with probability density function (pdf)

$$
g(y, \sigma)=\frac{e^{-\frac{y}{\sigma}}}{\sigma\left(1+e^{-\frac{y}{\sigma}}\right)^{2}}, \quad-\infty<y<\infty, \quad \sigma>0
$$

and cumulative distribution function (cdf)

$$
G(y, \sigma)=\frac{1}{1+e^{-\frac{y}{\sigma}}}, \quad-\infty<y<+\infty, \quad \sigma>0 .
$$

Suppose we observe $m$ upper record values $Y_{U(1)}=y_{1}, Y_{U(2)}=y_{2}, \ldots$, $Y_{U(m)}=y_{m}$ from the logistic distribution with pdf (1). The likelihood function (LF) of $\sigma$ for the given record sample $\mathbf{y}=\left(y_{1}, y_{2}, \ldots, y_{m}\right)$ is given (see Arnold et al., 1998) by

$$
\begin{aligned}
L(\sigma \mid \mathbf{y}) & =g\left(y_{m}, \sigma\right) \prod_{i=1}^{m-1} \frac{g\left(y_{i}, \sigma\right)}{1-G\left(y_{i}, \sigma\right)} \\
& =e^{-\frac{y_{m}}{\sigma}} \sigma^{-m} \frac{\prod_{i=1}^{m}\left(1+e^{\left.-\frac{y_{i}}{\sigma}\right)^{-1}}\right.}{1+e^{-\frac{y_{m}}{\sigma}}} .
\end{aligned}
$$

The log-likelihood function is

$$
\ln L(\sigma \mid \mathbf{y})=-\frac{y_{m}}{\sigma}-m \ln \sigma-\sum_{i=1}^{m} \ln \left(1+e^{-\frac{y_{i}}{\sigma}}\right)-\ln \left(1+e^{-\frac{y_{m}}{\sigma}}\right) .
$$


Taking the derivative with respect to $\sigma$ and equating it to zero, we obtain the likelihood equation for $\sigma$ as

$$
\begin{aligned}
\frac{d \ln L(\sigma \mid \mathbf{y})}{d \sigma} & =\frac{y_{m}}{\sigma^{2}}-\frac{m}{\sigma}-\frac{1}{\sigma^{2}} \sum_{i=1}^{m} \frac{y_{i} e^{-\frac{y_{i}}{\sigma}}}{1+e^{-\frac{y_{i}}{\sigma}}}-\frac{1}{\sigma^{2}} \frac{y_{m} e^{-\frac{y_{m}}{\sigma}}}{1+e^{-\frac{y_{m}}{\sigma}}} \\
& =0,
\end{aligned}
$$

or

$$
\frac{y_{m}}{\sigma}-\frac{1}{\sigma} \sum_{i=1}^{m} \frac{y_{i} e^{-\frac{y_{i}}{\sigma}}}{1+e^{-\frac{y_{i}}{\sigma}}}-\frac{1}{\sigma} \frac{y_{m} e^{-\frac{y_{m}}{\sigma}}}{1+e^{-\frac{y_{m}}{\sigma}}}-m=0 .
$$

Since (4) can not be solved analytically, some numerical methods such as Newton-Raphson method should be used to find the MLE of $\sigma$.

The following theorem shows the existence and uniqueness of the MLE of $\sigma$.

Theorem 1. Suppose we have observed the first $m$ upper record values $Y_{U(1)}=y_{1}, Y_{U(2)}=y_{2}, \ldots, Y_{U(m)}=y_{m}$ from the logistic distribution with $p d f(1)$. Then, the MLE of $\sigma$ exists and is unique if and only if $y_{m}>0$.

Proof. Define $\varphi(\sigma)=\frac{\sigma^{2} d \ln L(\sigma \mid \mathbf{y})}{d \sigma}$. We have

$$
\varphi(\sigma)=\frac{\sigma^{2} d \ln L(\sigma \mid \mathbf{y})}{d \sigma}=y_{m}-m \sigma-\sum_{i=1}^{m} \frac{y_{i} e^{-\frac{y_{i}}{\sigma}}}{1+e^{-\frac{y_{i}}{\sigma}}}-\frac{y_{m} e^{-\frac{y_{m}}{\sigma}}}{1+e^{-\frac{y_{m}}{\sigma}}} .
$$

We note that

$$
\lim _{\sigma \rightarrow 0} \varphi(\sigma)=y_{m}, \quad \lim _{\sigma \rightarrow+\infty} \varphi(\sigma)=-\infty
$$

and

$$
\varphi^{\prime}(\sigma)=-m-\sum_{i=1}^{m} \frac{y_{i}^{2} e^{-\frac{y_{i}}{\sigma}}}{\sigma^{2}}-\frac{y_{m}^{2} e^{-\frac{y_{m}}{\sigma}}}{\sigma^{2}}<0 .
$$

It follows that $\varphi(0)>0$ if and only if $y_{m}>0$. Therefore in this case, $\varphi(\sigma)$ is a continuous function on $(0, \infty)$ which decreases monotonically from positive to negative values. Therefore, the MLE of $\sigma$ which is a solution to $\varphi(\sigma)=0$, exists and is unique if and only if $y_{m}>0$.

Let us now discuss $P_{r}\left(Y_{U(m)}>0\right)$ for different values of $m$. The density function of the $m$ th upper record value $Y_{U(m)}$ is given (see Arnold et al., 1998) by

$$
f_{m}\left(y_{m}\right)=f\left(y_{m}\right) \frac{\left(-\ln \left[1-F\left(y_{m}\right)\right]\right)^{m}}{m !}, \quad-\infty<y_{m}<\infty .
$$


For the scaled logistic distribution, the density function of $Y_{U(m)}$ becomes

$$
f_{m}\left(y_{m}\right)=\frac{e^{-\frac{y_{m}}{\sigma}}}{\sigma\left(1+e^{\left.-\frac{y_{m}}{\sigma}\right)^{2}}\right.} \cdot \frac{\left(\frac{y_{m}}{\sigma}+\ln \left[1+e^{-\frac{y_{m}}{\sigma}}\right]\right)^{m}}{m !}, \quad-\infty<y_{m}<\infty .
$$

In Table 1, we have presented the values of $P_{r}\left(Y_{U(m)}>0\right)=\int_{0}^{\infty} f_{m}\left(y_{m}\right) d y_{m}$, for different values of $m$. From Table 1, we can see that $P_{r}\left(Y_{U(m)}>0\right)$ is very large. This means that the chances of obtaining the MLEs are very high in practice. Also, as the sample size $m$ increases, the probability of the feasibility of the MLE increases. On the other hand, since $P_{r}\left(Y_{U(m)}>0\right)=P_{r}\left(\frac{Y_{U(m)}}{\sigma}>0\right)$, the above probability does not depend on the parameter $\sigma$.

Table 1. Values of $P_{r}\left(Y_{U(m)}>0\right)$ for different $m$.

\begin{tabular}{cccccccc}
\hline \hline$m$ & 2 & 3 & 5 & 7 & 10 & 15 & 20 \\
\hline$P_{r}\left(Y_{U(m)}>0\right)$ & 0.9667 & 0.9944 & 0.9999 & 0.9999 & 0.9999 & 1.0000 & 1.0000 \\
\hline
\end{tabular}

\subsection{Approximate Maximum Likelihood Estimation}

Since the MLE of $\sigma$ when it exists, is not in an explicit form, we here propose the approximate MLE which has the explicit expression. Let us consider $x_{i}=\frac{y_{i}}{\sigma}, i=1,2, \ldots, m$. Then $x_{1}, \ldots, x_{m}$ are the first $m$ observed record data from the standard logistic distribution with pdf and cdf as

$$
f(x)=\frac{e^{-x}}{\left(1+e^{-x}\right)^{2}}, \quad-\infty<x<\infty,
$$

and

$$
F(x)=\frac{1}{1+e^{-x}}, \quad-\infty<x<\infty,
$$

respectively. Since $g(y, \sigma)=\frac{1}{\sigma} f\left(\frac{y}{\sigma}\right)$ and $G(y, \sigma)=F\left(\frac{y}{\sigma}\right)$, and

$$
f(x)=F(x)[1-F(x)], \quad f^{\prime}(x)=f(x)[1-2 F(x)],
$$

the LF based on the transformed record data, $\mathbf{x}=\left(x_{1}, \ldots, x_{m}\right)$ may be rewritten as

$$
L(\sigma) \equiv L(\sigma \mid \mathbf{x})=\sigma^{-m} f\left(x_{m}\right) \prod_{i=1}^{m-1} F\left(x_{i}\right) .
$$


The log-likelihood function is

$$
\ln L(\sigma)=-m \ln \sigma+\ln f\left(x_{m}\right)+\sum_{i=1}^{m-1} \ln F\left(x_{i}\right) .
$$

By using the equation (6), we derive the likelihood equation for $\sigma$ from (8), as

$$
\frac{d \ln L(\sigma)}{d \sigma}=-\frac{1}{\sigma}\left\{m+\sum_{i=1}^{m} x_{i}-x_{m} F\left(x_{m}\right)-\sum_{i=1}^{m} x_{i} F\left(x_{i}\right)\right\}=0 .
$$

The likelihood equation in (9) does not admit an explicit solution because of the presence of the term $F\left(x_{i}\right) i=1, \ldots, m$. Therefore, we approximate the term $F\left(x_{i}\right)$ by expanding it in a Taylor series around $E\left(X_{i}\right)=\delta_{i}$. Some approximate solutions for the MLEs have been discussed in the book by Tiku et al. (1986). Balakrishnan and Aggarwala (2000), Balakrishnan and Kannan (2000), Balakrishnan and Asgharzadeh (2005), Raqab et al. (2010) and Asgharzadeh et al. (2011) used approximate solutions for the MLEs when the data are progressively censored.

From Arnold et al. (1998), it is known that

$$
F\left(X_{i}\right) \stackrel{d}{=} U_{i},
$$

where $U_{i}$ is the $i$ th record statistic from the uniform $U(0,1)$ distribution. We then have

$$
X_{i} \stackrel{d}{=} F^{-1}\left(U_{i}\right)
$$

and hence

$$
\delta_{i}=E\left(X_{i}\right) \approx F^{-1}\left(E\left(U_{i}\right)\right) .
$$

From Arnold et al. (1998), it is known that

$$
E\left(U_{i}\right)=1-\left(\frac{1}{2}\right)^{i+1}, \quad i=1, \ldots, m .
$$

Since, for the standard logistic distribution, we have

$$
F^{-1}(u)=\ln \left(\frac{u}{1-u}\right)
$$

we can approximate $\delta_{i}$ by $F^{-1}\left\{1-\left(\frac{1}{2}\right)^{i+1}\right\}=\ln \left(2^{i+1}-1\right)$. 
Now, upon expanding the function $F\left(x_{i}\right)$ around the point $\delta_{i}$ and keeping only the first two terms, we get

$$
\begin{aligned}
F\left(x_{i}\right) & \simeq F\left(\delta_{i}\right)+\left(x_{i}-\delta_{i}\right) f\left(\delta_{i}\right) \\
& =\alpha_{i}+\beta_{i} x_{i}, \quad i=1, \ldots, m,
\end{aligned}
$$

where

$$
\alpha_{i}=F\left(\delta_{i}\right)-\delta_{i} f\left(\delta_{i}\right), \quad i=1, \ldots, m,
$$

and

$$
\beta_{i}=f\left(\delta_{i}\right) \geqslant 0, \quad i=1, \ldots, m .
$$

Using the expression in (14), we approximate the likelihood equation in (9) by

$$
\frac{d \ln L^{*}(\sigma)}{d \sigma}=-\frac{1}{\sigma}\left\{m+\sum_{i=1}^{m} x_{i}-x_{m}\left(\alpha_{m}+\beta_{m} x_{m}\right)-\sum_{i=1}^{m} x_{i}\left(\alpha_{i}+\beta_{i} x_{i}\right)\right\}=0
$$

which can be rewritten as

$$
-m-\frac{1}{\sigma}\left(\sum_{i=1}^{m} y_{i}-\alpha_{m} y_{m}-\sum_{i=1}^{m} \alpha_{i} y_{i}\right)+\frac{1}{\sigma^{2}}\left(\beta_{m} y_{m}^{2}+\sum_{i=1}^{m} \beta_{i} y_{i}^{2}\right)=0
$$

By solving the quadratic equation in (16) for $\sigma$, we obtain the approximate MLE of $\sigma$ as

$$
\widetilde{\sigma}=\frac{-A+\sqrt{A^{2}+4 m B}}{2 m},
$$

where

$$
A=\sum_{i=1}^{m} y_{i}-\alpha_{m} y_{m}-\sum_{i=1}^{m} \alpha_{i} y_{i}, \quad B=\beta_{m} y_{m}^{2}+\sum_{i=1}^{m} \beta_{i} y_{i}^{2} .
$$

Note that Eq. (16) has two roots but since $B \geqslant 0$, only one root in (17) is admissible. The approximate MLE in (17) may provide us with a good starting value for the iterative solution of the likelihood equation in (4).

\subsection{Bayes Estimation}

Here we consider the Bayes estimation of the unknown parameter $\sigma$. The loss function is squared error. It is assumed that $\sigma$ has an inverse gamma prior, $I G(a, b)$, with pdf

$$
\pi(\sigma) \propto e^{-\frac{b}{\sigma}} \sigma^{-(a+1)}, \quad \sigma>0, \quad a, b>0 .
$$


This prior is suitable for deriving the posterior density. If $a=b=0$, the prior has the pdf

$$
\pi(\sigma) \propto \frac{1}{\sigma},
$$

which is the Jeffreys non-informative prior distribution of the parameter $\sigma$. The inverse gamma prior distribution is widely used in Bayesian analysis. It is often used as the conjugate prior of the variance parameter in a normal distribution. It is also used as the conjugate prior of the exponential mean in a exponential distribution.

To choose the hyper-parameters $a$ and $b$ in (19), several methods have been discussed in the literature. As suggested by Berger (1980), a common way for determining prior parameters is to calculate them from estimated prior moments. For the inverse gamma prior distribution, one can specify the prior mean and variance for $\sigma$, and then use the relationships

$$
E(\sigma)=\frac{b}{a-1}, \quad a>1, \quad \operatorname{Var}(\sigma)=\frac{b^{2}}{(a-1)^{2}(a-2)}, \quad a>2
$$

to determine $a$ and $b$.

By combining the LF in (3) and the prior pdf in (19), we obtain the posterior density of $\sigma$ as

$$
\pi(\sigma \mid \mathbf{y}) \propto e^{-\frac{1}{\sigma}\left(y_{m}+b\right)} \sigma^{-(m+a+1)} \frac{\prod_{i=1}^{m}\left(1+e^{\left.-\frac{y_{i}}{\sigma}\right)^{-1}}\right.}{1+e^{-\frac{y_{m}}{\sigma}}} .
$$

The Bayes estimator of $\sigma$ under squared error loss function is the posterior mean of $\sigma$ which can be written as

$$
\hat{\sigma}_{B S}=\frac{\int_{0}^{\infty} \sigma \pi(\sigma \mid \mathbf{y}) d \sigma}{\int_{0}^{\infty} \pi(\sigma \mid \mathbf{y}) d \sigma} .
$$

It is clear that the Bayes estimator of $\sigma$ can not be obtained in a closed form, due to the complex form of $\pi(\sigma \mid \mathbf{y})$. Here we use importance sampling scheme to obtain the Bayes estimator of $\sigma$ as follows. Note that the posterior density of $\sigma$ can be written as

$$
\pi(\sigma \mid \mathbf{y}) \propto I G\left(m+a, y_{m}+b\right) h(\sigma)
$$

where

$$
h(\sigma)=\frac{\prod_{i=1}^{m}\left(1+e^{\left.-\frac{y_{i}}{\sigma}\right)^{-1}}\right.}{1+e^{-\frac{y_{m}}{\sigma}}} .
$$


Substituting (21) in (20), we can obtain the Bayes estimator of $\sigma$ as

$$
\hat{\sigma}_{B S}=\frac{E_{\sigma}[\sigma h(\sigma)]}{E_{\sigma}[h(\sigma)]},
$$

where $E$ denotes the expectation with respect to the inverse gamma $I G(m+$ $\left.a, y_{m}+b\right)$.

We now apply importance sampling scheme to generate samples from the posterior distribution $\pi(\sigma \mid \mathbf{x})$. The steps are:

Step 1. Generate $\sigma$ from $I G\left(m+a, y_{m}+b\right)$.

Step 2. Repeat Step 1, M times to obtain $\sigma_{1}, \ldots, \sigma_{M}$.

Now, using (23), an approximate Bayesian estimate of $\sigma$ can obtained as

$$
\hat{\hat{\sigma}}_{B S}=\frac{\frac{1}{M} \sum_{j=1}^{M} \sigma_{j} h\left(\sigma_{j}\right)}{\frac{1}{M} \sum_{j=1}^{M} h\left(\sigma_{j}\right)} .
$$

For more details on important sampling scheme, see Robert and Casella (2004).

\section{Confidence Intervals}

In this section, we propose different confidence intervals of the unknown parameter $\sigma$.

\subsection{Approximate Confidence Interval}

In mathematical statistics, the quantity $E\left[\frac{d \ln L(\sigma)}{d \sigma}\right]^{2}$ is called the Fisher information of the sample about $\sigma$, and will be denoted by $I(\sigma)$. Under some regularity conditions (e.g., see Ferguson, 1996, pp. 121), it can be shown that $I(\sigma)=-E\left[\frac{d^{2} \ln L(\sigma)}{d \sigma^{2}}\right]$.

First, let us derive the Fisher information based on the likelihood as well as the approximate likelihood functions. From the log-likelihood function in (9), we obtain

$$
\begin{aligned}
\frac{d^{2} \ln L(\sigma)}{d \sigma^{2}}= & \frac{m}{\sigma^{2}}+\frac{2 \sum_{i=1}^{m} x_{i}\left\{1-F\left(x_{i}\right)\right\}}{\sigma^{2}}-\frac{2 x_{m} F\left(x_{m}\right)}{\sigma^{2}} \\
& -\frac{\sum_{i=1}^{m} x_{i}^{2} f\left(x_{i}\right)}{\sigma^{2}}-\frac{x_{m}^{2} f\left(x_{m}\right)}{\sigma^{2}} .
\end{aligned}
$$


From this expression, we obtain the Fisher information as

$$
\begin{aligned}
I(\sigma)= & -E\left\{\frac{d^{2} \ln L(\sigma)}{d \sigma^{2}}\right\} \\
= & -\frac{m}{\sigma^{2}}-\frac{2 \sum_{i=1}^{m} E\left[X_{i}\left\{1-F\left(X_{i}\right)\right\}\right]}{\sigma^{2}}+\frac{2 E\left[X_{m} F\left(X_{m}\right)\right]}{\sigma^{2}} \\
& +\frac{\sum_{i=1}^{m} E\left\{X_{i}^{2} f\left(X_{i}\right)\right\}}{\sigma^{2}}+\frac{E\left\{X_{m}^{2} f\left(X_{m}\right)\right\}}{\sigma^{2}} .
\end{aligned}
$$

Proceeding similarly, we obtain from the approximate likelihood equation in (15) that

$$
\begin{aligned}
\frac{d^{2} \ln L^{*}(\sigma)}{d \sigma^{2}}= & \frac{m}{\sigma^{2}}+\frac{2 \sum_{i=1}^{m}\left(1-\alpha_{i}\right) x_{i}}{\sigma^{2}}-\frac{2 \alpha_{m} x_{m}}{\sigma^{2}}-\frac{3 \sum_{i=1}^{m} \beta_{i} x_{i}^{2}}{\sigma^{2}} \\
& -\frac{3 \beta_{m} x_{m}^{2}}{\sigma^{2}} .
\end{aligned}
$$

From this expression, we obtain the Fisher information as

$$
\begin{aligned}
I^{*}(\sigma)= & -E\left\{\frac{d^{2} \ln L^{*}(\sigma)}{d \sigma^{2}}\right\} \\
= & -\frac{m}{\sigma^{2}}-\frac{2 \sum_{i=1}^{m}\left(1-\alpha_{i}\right) E\left(X_{i}\right)}{\sigma^{2}}+\frac{2 \alpha_{m} E\left(X_{m}\right)}{\sigma^{2}}+\frac{3 \sum_{i=1}^{m} \beta_{i} E\left(X_{i}^{2}\right)}{\sigma^{2}} \\
& +\frac{3 \beta_{m} E\left(X_{m}^{2}\right)}{\sigma^{2}} .
\end{aligned}
$$

To derive the Fisher information in (26) and (28), we first need to calculate the four expectations $E\left[X_{i}\right], E\left[X_{i}^{2}\right], E\left\{X_{i} F\left(X_{i}\right)\right\}$ and $E\left\{X_{i}^{2} f\left(X_{i}\right)\right\}$. From Ahsanullah (1995), we have

$$
E\left[X_{1}\right]=0, \quad E\left[X_{i}\right]=\sum_{l=2}^{i} \zeta(l), \quad i \geqslant 2,
$$

and

$$
E\left[X_{i}^{2}\right]=2 i \sum_{l=2}^{i+1} \zeta(l)-i(i+1)+\sum_{l=2}^{\infty} \frac{B_{l}}{(l+1)^{i}}
$$

where $\zeta(\cdot)$ is Riemann zeta function $\zeta(n)=\sum_{k=1}^{\infty} k^{-n}$ and for $n \geqslant 2$

$$
B_{n}=\frac{1}{n}\left(1+\frac{1}{2}+\cdots+\frac{1}{n-1}\right) \text {. }
$$


Also, for $i \geqslant 1$, we have

$$
E\left[X_{i}\left\{1-F\left(X_{i}\right)\right\}\right]=\frac{i}{2^{i+1}}-\sum_{l=1}^{\infty} \frac{1}{l(l+2)^{i}}
$$

and

$$
\begin{aligned}
E\left[X_{i}^{2} F\left(X_{i}\right)\left\{1-F\left(X_{i}\right)\right\}\right]= & \sum_{l=1}^{\infty}\left\{\frac{1}{l^{2}(2 l+2)^{i}}-\frac{1}{l^{2}(2 l+3)^{i}}\right\} \\
& +2 \sum_{1 \leqslant l<k<\infty}\left\{\frac{1}{l k(l+k+2)^{i}}-\frac{1}{l k(l+k+3)^{i}}\right\} \\
& +2 i \sum_{l=1}^{\infty}\left\{\frac{1}{l(l+3)^{i+1}}-\frac{1}{l(l+2)^{i+1}}\right\} \\
& +i(i+1)\left(\frac{1}{2^{i+2}}-\frac{1}{3^{i+2}}\right) .
\end{aligned}
$$

For the proofs of (29) and (30), see the Appendix. Moreover, $E\left\{X_{i} F\left(X_{i}\right)\right\}$ and $E\left\{X_{i}^{2} f\left(X_{i}\right)\right\}$ can be obtained from the expressions

$$
\begin{aligned}
& E\left\{X_{i} F\left(X_{i}\right)\right\}=E\left[X_{i}\right]-E\left[X_{i}\left\{1-F\left(X_{i}\right)\right\}\right] \\
& E\left\{X_{i}^{2} f\left(X_{i}\right)\right\}=E\left[X_{i}^{2} F\left(X_{i}\right)\left\{1-F\left(X_{i}\right)\right\}\right],
\end{aligned}
$$

and using the above expectations.

Now under the regularity conditions, the variance of the MLE, $\widehat{\sigma}$, can be approximated by inverting the Fisher information, i.e, $\widehat{\operatorname{Var}}(\widehat{\sigma}) \approx I^{-1}(\widehat{\sigma})$. Then, the approximate $100(1-\gamma) \%$ confidence interval for $\sigma$ based on MLE is given by

$$
\left(\widehat{\sigma} \pm z_{\frac{\gamma}{2}} \sqrt{\widehat{\operatorname{Var}}(\widehat{\sigma})}\right)
$$

Similar approximate confidence interval can be obtained based on AMLE also.

\subsection{Bootstrap Confidence Intervals}

In this subsection, two parametric bootstrap procedures are proposed to construct the bootstrap confidence intervals. The first one is the percentile bootstrap (Boot-p) confidence interval proposed by Efron (1982). The second one is the bootstrap-t (Boot-t) confidence interval proposed by Hall 
(1988). These two parametric bootstrap procedures are described as follows.

\section{Boot-p Procedure:}

1. Estimate $\sigma$, say $\widehat{\sigma}$, from sample based on the MLE procedure.

2. Generate a bootstrap sample $\left\{X_{1}^{*}, \ldots, X_{m}^{*}\right\}$, using $\widehat{\sigma}$. Obtain the bootstrap estimate of $\sigma$, say $\widehat{\sigma}^{*}$ using the bootstrap sample.

3. Repeat Step 2 NBOOT times.

4. Order $\widehat{\sigma}_{1}^{*}, \ldots, \widehat{\sigma}_{N B O O T}^{*}$ as $\widehat{\sigma}_{(1)}^{*}, \ldots, \widehat{\sigma}_{(N B O O T)}^{*}$. Then, the approximate $100(1-\gamma) \%$ confidence interval for $\sigma$ becomes

$$
\left(\widehat{\sigma^{*}} \text { Boot-p }\left(\frac{\gamma}{2}\right), \widehat{\sigma}^{*} \text { Boot-p }\left(1-\frac{\gamma}{2}\right)\right)
$$

\section{Boot-t Procedure:}

1. Estimate $\sigma$, say $\widehat{\sigma}$, from sample based on the MLE procedure.

2. Generate a bootstrap sample $\left\{X_{1}^{*}, \ldots, X_{m}^{*}\right\}$, using $\widehat{\sigma}$ and obtain the bootstrap estimate of $\sigma$, say $\widehat{\sigma}^{*}$. Also compute $\widehat{V}\left(\widehat{\sigma^{*}}\right)=I^{-1}\left(\widehat{\sigma^{*}}\right)$.

3. Determine the $T^{*}$ statistic

$$
T^{*}=\frac{\left(\widehat{\sigma^{*}}-\widehat{\sigma}\right)}{\sqrt{\widehat{V}\left(\widehat{\sigma^{*}}\right)}} .
$$

4. Repeat Steps 2 and 3 NBOOT times.

5. Define $\widehat{\sigma}_{\text {Boot }-t}^{*}=\widehat{\sigma}+\sqrt{\widehat{V}\left(\widehat{\left.\sigma^{*}\right)}\right.} T^{*}$. Order $\widehat{\sigma}_{1}^{*}, \ldots, \widehat{\sigma}_{N B O O T}^{*}$ as $\widehat{\sigma}_{(1)}^{*}, \ldots$, $\widehat{\sigma}_{(N B O O T)}^{*}$. Then, the approximate $100(1-\gamma) \%$ confidence interval for $\sigma$ becomes

$$
\left({\widehat{\sigma^{*}}}_{\text {Boot }-t}\left(\frac{\gamma}{2}\right),{\widehat{\sigma^{*}}}_{\text {Boot }-t}\left(1-\frac{\gamma}{2}\right)\right)
$$




\subsection{Credible Interval}

In Bayesian statistics, we use the posterior distribution to determine Bayesian confidence intervals or credible intervals. An interval $\left(I_{1}, I_{2}\right)$ is a $100(1-\gamma) \%$ credible interval for $\sigma$ if

$$
\int_{I_{1}}^{I_{2}} \pi(\sigma \mid \mathbf{y}) d \sigma=1-\gamma
$$

Credible intervals are not unique. We can form a credible interval by taking the highest posterior density (HPD) interval of the parameter space. A HPD interval is such that the posterior density for every point inside the interval is greater than that for every point outside of it.

Here we obtain the credible interval of $\sigma$ using the idea of Chen and Shao (1999). Consider the posterior density of $\sigma, \pi(\sigma \mid \mathbf{y})$. Let us denote $\Pi(\sigma \mid \mathbf{y})$ as the posterior distribution function of $\sigma$. Denote $\sigma^{(\gamma)}$ as the $\gamma$ th quantile of $\sigma$ where $\sigma^{(\gamma)}=\inf \{\sigma: \Pi(\sigma \mid \mathbf{x}) \geqslant \gamma\}$ and $0<\gamma<1$. For a given $\sigma^{*}$, we have $\Pi\left(\sigma^{*} \mid \mathbf{y}\right)=E\left[I_{\sigma \leqslant \sigma^{*}}(\sigma) \mid \mathbf{y}\right]$, where $I_{\sigma \leqslant \sigma^{*}}(\sigma)$ is the indicator function defined by

$$
I_{\sigma \leqslant \sigma^{*}}(\sigma)= \begin{cases}1 & \text { if } \sigma \leqslant \sigma^{*} \\ 0 & \text { if } \sigma>\sigma^{*},\end{cases}
$$

hence, a simulation consistent estimator of $\Pi\left(\sigma^{*} \mid \mathbf{y}\right)$ is

$$
\hat{\Pi}\left(\sigma^{*} \mid \mathbf{y}\right)=\frac{\sum_{i=1}^{M} I_{\sigma \leqslant \sigma^{*}}(\sigma) h\left(\sigma_{i}\right)}{\sum_{i=1}^{M} h\left(\sigma_{i}\right)}
$$

where $h(\cdot)$ is given in $(22)$.

Now, suppose that $\sigma_{(1)}, \ldots, \sigma_{(n)}$ are the ordered values of $\sigma_{1}, \ldots, \sigma_{n}$. Define

$$
w_{i}=\frac{h\left(\sigma_{(i)}\right)}{\sum_{i=1}^{M} h\left(\sigma_{(i)}\right)} \quad i=1,2, \ldots, M .
$$

Then, a simulation consistent estimator of $\Pi\left(\sigma^{*} \mid \mathbf{y}\right)$ is given by

$$
\widehat{\Pi}\left(\sigma^{*} \mid \mathbf{y}\right)=\left\{\begin{array}{lll}
0 & \text { if } & \sigma^{*}<\sigma_{(1)} \\
\sum_{j=1}^{i} w_{j} & \text { if } & \sigma_{(i)} \leqslant \sigma^{*}<\sigma_{(i+1)} \\
1 & \text { if } & \sigma^{*} \geqslant \sigma_{(M)} .
\end{array}\right.
$$

Thus, an approximate estimate of $\sigma^{(\gamma)}$ is obtained as

$$
\hat{\sigma}^{(\gamma)}= \begin{cases}\sigma_{(1)} & \text { if } \quad \gamma=0 \\ \sigma_{(i)} & \text { if } \quad \sum_{j=1}^{i-1} w_{j}<\gamma \leqslant \sum_{j=1}^{i} w_{j} .\end{cases}
$$


Now let us consider the following intervals

$$
\left(\hat{\sigma}^{\left(\frac{i}{M}\right)}, \hat{\sigma}^{\left(\frac{i+[(1-\gamma) M]}{M}\right)}\right), \quad i=1,2, \ldots, M-[(1-\gamma) M]
$$

where $[a]$ denotes the greatest integer less than or equal to $a$. We then choose the interval with the smallest width. This interval will be the $100(1-\gamma) \%$ HPD interval estimate of $\sigma$.

\section{Numerical Computations}

In this section, one numerical example and a Monte Carlo simulation are presented to illustrate all the estimation methods described in the preceding sections.

\section{Example 1 (Real data set).}

The following data are the amount of annual maximum daily rainfall at Mehrabad Synoptic Station from 1952 to 2000 (see the website: http://www.iranhydrology.com/meteo.asp)

$\begin{array}{llllllllll}19 & 20 & 22 & 16 & 13 & 35 & 24 & 20 & 28 & 13 \\ 48 & 15 & 16 & 22 & 32 & 10 & 15 & 34 & 31 & 16 \\ 33 & 28 & 20 & 26 & 19 & 23 & 22 & 35 & 16 & 24 \\ 38 & 26 & 22 & 21 & 26 & 29 & 25 & 22 & 22.2 & 47 \\ 23.1 & 23 & 39 & 32.1 & 49 & 24.2 & 26 & 18.2 & 28 . & \end{array}$

After subtracting 25 from the above data, we analysed the transformed data set by using the logistic distribution with scale parameter 4.851. It is observed that the Kolmogorov-Smirnov (K-S) distance and the corresponding $\mathrm{P}$-value are respectively

$$
\mathrm{K}-\mathrm{S}=0.1221, \quad \text { and } \quad \mathrm{p} \text {-value }=0.4335 .
$$

Hence the logistic model (1) fits quite well to the transformed data.

For the transformed data, we observe the following six upper record values:

$$
\begin{array}{llllll}
-6 & -5 & -3 & 10 & 23 & 24 .
\end{array}
$$

Note that for the above record data, the K-S distance and the corresponding $\mathrm{P}$-value are respectively

$$
\mathrm{K}-\mathrm{S}=0.3677, \quad \text { and } \quad \mathrm{p} \text {-value }=0.3137,
$$


which supports conclusion above.

Here, we have $m=6, A=-23.734$ and $B=30.105$. From (18), we obtain the AMLE of $\sigma$ as

$$
\tilde{\sigma}=\frac{-A+\sqrt{A^{2}+4 m B}}{2 m}=4.966 .
$$

The MLE of $\sigma$ is 5.316. Note that the MLE was obtained by solving the nonlinear equation (4), in which the AMLE was used as the starting value for the iterations. The Bayes estimator of $\sigma$ is 4.762. To compute the Bayes estimators, since we do not have any prior information, we assumed that $a=b=0$. In the case, the prior distribution for $\sigma$ becomes improper. We also computed $95 \%$ confidence intervals for $\sigma$. The approximate confidence intervals based MLE and AMLE are (1.510, 9.123) and (2.016, 7.916), respectively. The p-boot and t-boot confidence intervals are $(2.258,10.030)$ and $(2.378,8.097)$. The HPD interval is $(3.074,11.807)$.

Let us now compare different estimation methods using a Monte Carlo simulation. In this simulation, we have randomly generated 1000 upper record sample $y_{1}, \ldots, y_{m}$ from the logistic distribution in (1). We also used two parameter values $\sigma=1,2$. In any replication if $y_{m}<0$, we have not considered that sample. We then obtained different point and interval estimators based on the methods described in Sections 2 and 3. We then compared the performances of the different point estimators MLE, AMLE and the Bayes estimator in terms of biases, and mean square errors (MSEs).

For computing Bayesian point and interval estimators, we use two priors as follows:

$$
\begin{array}{ll}
\text { Prior 1: } & a=0, \quad b=0 \\
\text { Prior 2: } & a=2.5, \quad b=1 .
\end{array}
$$

Note that Prior 1 is the non-informative inverse gamma prior for $\sigma$. But Prior 2 is an informative inverse gamma prior in which the hyper-parameters $a$ and $b$ have been chosen such that we have $E(\sigma)=\frac{1}{2}$ and $\operatorname{Var}(\sigma)=\frac{1}{4}$.

For various choices of $m$, Table 2 presents the average biases, and MSEs of different point estimators from this simulation study. All the computations are performed using the SPLUS package.

From Table 2, we observe that the MLE and the AMLE are almost identical in terms of both biases and MSEs. Comparing the two Bayesan estimators based on two Priors 1 and 2 clearly shows that the Bayesian estimators 
based on Prior 2 work better than the Bayesian estimatorts based on noninformative Prior 1, in terms of both biases and MSEs. It is also observed that if we have informative priors, Bayesian estimators have a clear advantage over the classical estimators MLE and AMLE. It is also observed as $m$ increases, the performances become better in terms of biases and MSEs in all cases considered.

Table 2. Biases and MSEs of Bayesian point estimators for different $m$ and $\sigma=1,2$.

\begin{tabular}{|c|c|c|c|c|c|c|c|c|c|}
\hline & & \multicolumn{4}{|c|}{$\sigma=1$} & \multicolumn{4}{|c|}{$\sigma=2$} \\
\hline & & \multirow{2}{*}{ MLE } & \multirow{2}{*}{ AMLE } & \multicolumn{2}{|c|}{ SEP } & \multirow{2}{*}{ MLE } & \multirow{2}{*}{ AMLE } & \multicolumn{2}{|c|}{ SEP } \\
\hline & & & & Prior 1 & Prior 2 & & & Prior 1 & Prior 2 \\
\hline \multirow[t]{2}{*}{$m=2$} & Bias & 0.562 & 0.567 & 0.763 & 0.464 & 1.441 & 1.536 & 2.303 & 1.355 \\
\hline & MSE & 0.316 & 0.318 & 0.556 & 0.232 & 2.076 & 2.359 & 3.304 & 1.918 \\
\hline \multirow[t]{2}{*}{$m=3$} & Bias & 0.401 & 0.416 & 0.645 & 0.352 & 1.206 & 1.319 & 1.625 & 1.181 \\
\hline & MSE & 0.281 & 0.296 & 0.402 & 0.192 & 1.879 & 1.955 & 2.446 & 1.502 \\
\hline \multirow[t]{2}{*}{$m=5$} & Bias & 0.322 & 0.338 & 0.556 & 0.241 & 0.584 & 0.663 & 0.850 & 0.458 \\
\hline & MSE & 0.104 & 0.115 & 0.314 & 0.058 & 0.341 & 0.382 & 0.701 & 0.210 \\
\hline \multirow[t]{2}{*}{$m=10$} & Bias & 0.266 & 0.289 & 0.436 & 0.181 & 0.480 & 0.507 & 0.733 & 0.376 \\
\hline & MSE & 0.071 & 0.084 & 0.190 & 0.033 & 0.262 & 0.286 & 0.452 & 0.157 \\
\hline \multirow[t]{2}{*}{$m=15$} & Bias & 0.182 & 0.201 & 0.341 & 0.171 & 0.294 & 0.332 & 0.421 & 0.219 \\
\hline & MSE & 0.060 & 0.071 & 0.111 & 0.025 & 0.109 & 0.126 & 0.219 & 0.067 \\
\hline \multirow[t]{2}{*}{$m=20$} & Bias & 0.075 & 0.087 & 0.183 & 0.048 & 0.153 & 0.168 & 0.250 & 0.106 \\
\hline & MSE & 0.029 & 0.036 & 0.066 & 0.012 & 0.074 & 0.088 & 0.113 & 0.039 \\
\hline
\end{tabular}

We also compared different confidence intervals (CIs) in terms of the average confidence lengths, and coverage percentages in Table 3 . The nominal level for the confidence intervals is 0.95 in each case.

From Table 3, we observe that the Boot-t CIs and HPD credible intervals based on informative Prior 2 provide the shortest confidence lengths. It is also observed that Boot-t CIs perform better than the Boot-p CIs. The HPD credible intervals based on non-informative Prior 1 do not work well. From Table 3, it is clear that the Approximate CIs based on AMLE and Boot$\mathrm{p}$ credible intervals provide the most coverage percentages in the most of cases considered. It is also noted that as $m$ increases, the average confidence lengths decrease in the most of cases considered. 
Table 3. Average confidence/credible length and coverage percentage for different $m$ and $\sigma=1,2$.

\begin{tabular}{|c|c|c|c|c|c|c|c|c|}
\hline & & & \multirow{2}{*}{ AMLE } & \multirow{2}{*}{ MLE } & \multirow{2}{*}{ p-boot } & \multirow{2}{*}{ t-boot } & \multicolumn{2}{|c|}{ SEP } \\
\hline & & & & & & & Prior 1 & Prior 2 \\
\hline \multirow{12}{*}{$\sigma=1$} & \multirow[t]{2}{*}{$m=2$} & Length & 2.223 & 1.794 & 1.984 & 1.226 & 3.226 & 1.345 \\
\hline & & Cov. Prob. & 0.824 & 0.777 & 0.835 & 0.655 & 0.673 & 0.685 \\
\hline & \multirow[t]{2}{*}{$m=3$} & Length & 2.002 & 1.628 & 1.818 & 1.190 & 2.760 & 1.205 \\
\hline & & Cov. Prob. & 0.896 & 0.862 & 0.895 & 0.745 & 0.727 & 0.738 \\
\hline & \multirow[t]{2}{*}{$m=5$} & Length & 1.917 & 1.568 & 1.775 & 1.158 & 2.281 & 1.135 \\
\hline & & Cov. Prob. & 0.824 & 0.783 & 0.837 & 0.745 & 0.719 & 0.683 \\
\hline & \multirow{2}{*}{$m=10$} & Length & 1.131 & 1.023 & 1.067 & 0.792 & 1.075 & 0.828 \\
\hline & & Cov. Prob. & 0.905 & 0.885 & 0.927 & 0.786 & 0.802 & 0.739 \\
\hline & \multirow[t]{2}{*}{$m=15$} & Length & 0.962 & 0.903 & 0.904 & 0.698 & 0.865 & 0.695 \\
\hline & & Cov. Prob. & 0.928 & 0.924 & 0.926 & 0.826 & 0.804 & 0.765 \\
\hline & \multirow[t]{2}{*}{$m=20$} & Length & 0.725 & 0.693 & 0.704 & 0.651 & 0.810 & 0.648 \\
\hline & & Cov. Prob. & 0.937 & 0.933 & 0.940 & 0.911 & 0.893 & 0.827 \\
\hline \multirow{12}{*}{$\sigma=2$} & \multirow[t]{2}{*}{$m=2$} & Length & 4.537 & 3.674 & 4.044 & 2.504 & 4.452 & 2.270 \\
\hline & & Cov. Prob. & 0.820 & 0.775 & 0.835 & 0.655 & 0.673 & 0.545 \\
\hline & \multirow[t]{2}{*}{$m=3$} & Length & 3.813 & 3.101 & 3.495 & 2.292 & 5.507 & 2.026 \\
\hline & & Cov. Prob. & 0.876 & 0.781 & 0.819 & 0.675 & 0.685 & 0.565 \\
\hline & \multirow[t]{2}{*}{$m=5$} & Length & 3.010 & 2.549 & 2.769 & 1.944 & 3.674 & 1.940 \\
\hline & & Cov. Prob. & 0.877 & 0.846 & 0.875 & 0.754 & 0.785 & 0.695 \\
\hline & \multirow[t]{2}{*}{$m=10$} & Length & 2.238 & 2.022 & 2.111 & 1.579 & 2.175 & 1.522 \\
\hline & & Cov. Prob. & 0.908 & 0.884 & 0.887 & 0.815 & 0.745 & 0.705 \\
\hline & \multirow[t]{2}{*}{$m=15$} & Length & 1.932 & 1.821 & 1.812 & 1.391 & 1.692 & 1.339 \\
\hline & & Cov. Prob. & 0.914 & 0.916 & 0.928 & 0.837 & 0.775 & 0.739 \\
\hline & \multirow[t]{2}{*}{$m=20$} & Length & 1.718 & 1.662 & 1.638 & 1.554 & 1.985 & 1.536 \\
\hline & & Cov. Prob. & 0.927 & 0.935 & 0.942 & 0.904 & 0.842 & 0.827 \\
\hline
\end{tabular}

Let us now consider a sequence of priors in order to perform a sensitive analysis. In Table 4, we provided the value of Bayesian point estimators for different values of $a$ and $b$. In Table 5 , we also provided the average confidence length and coverage percentage of different CIs for different $a$ and $b$. From Tables 4 and 5 , it is clear that the results are not changed significantly by changing the prior parameters $a$ and $b$. From Tables for $b$ fixed, when $a$ is increasing, the biases, MSEs and the average confidence lengths decrease, which is reasonable. Since in this case, the variance of the prior distribution decreases and hence, the efficiency of the prior increases. 
Table 4. Biases and MSEs of Bayes estimators for different $m$, and different values of $a$ and $b$.

\begin{tabular}{|c|c|c|c|c|c|c|c|c|}
\hline & & & \multicolumn{3}{|c|}{$a=3$} & \multicolumn{3}{|c|}{$b=3$} \\
\hline & & & $b=2$ & $b=3$ & $b=4$ & $a=1$ & $a=2$ & $a=4$ \\
\hline \multirow{6}{*}{$\sigma=1$} & \multirow[t]{2}{*}{$m=3$} & Bias & 0.342 & 0.376 & 0.331 & 0.516 & 0.466 & 0.417 \\
\hline & & MSE & 0.215 & 0.246 & 0.277 & 0.302 & 0.275 & 0.251 \\
\hline & \multirow{2}{*}{$m=5$} & Bias & 0.229 & 0.238 & 0.249 & 0.430 & 0.449 & 0.391 \\
\hline & & MSE & 0.163 & 0.140 & 0.119 & 0.219 & 0.197 & 0.158 \\
\hline & \multirow[t]{2}{*}{$m=10$} & Bias & 0.173 & 0.195 & 0.212 & 0.327 & 0.316 & 0.283 \\
\hline & & MSE & 0.073 & 0.091 & 0.109 & 0.133 & 0.115 & 0.090 \\
\hline \multirow{6}{*}{$\sigma=2$} & \multirow[t]{2}{*}{$m=3$} & Bias & 1.089 & 1.037 & 1.094 & 1.041 & 0.920 & 0.952 \\
\hline & & MSE & 0.839 & 0.874 & 0.914 & 1.021 & 0.938 & 0.894 \\
\hline & \multirow[t]{2}{*}{$m=5$} & Bias & 0.839 & 0.860 & 0.819 & 0.810 & 0.788 & 0.765 \\
\hline & & MSE & 0.628 & 0.662 & 0.683 & 0.704 & 0.673 & 0.633 \\
\hline & \multirow[t]{2}{*}{$m=10$} & Bias & 0.311 & 0.295 & 0.281 & 0.362 & 0.311 & 0.277 \\
\hline & & MSE & 0.329 & 0.362 & 0.380 & 0.397 & 0.361 & 0.307 \\
\hline
\end{tabular}

Table 5. Average credible length and coverage percentage of Bayes estimations for different $m$.

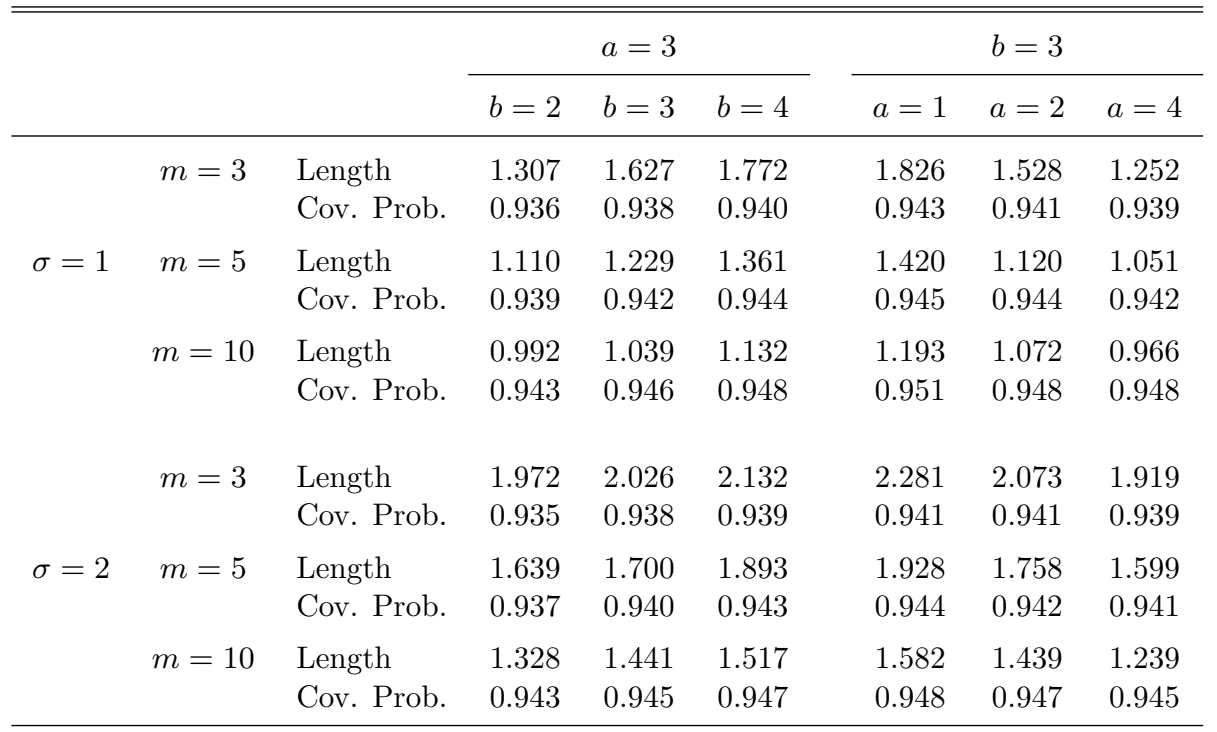




\section{References}

Ahmadi, J. and Arghami, N.R. (2001). On the Fisher Information in Record Values. Metrika, 53, 195-206.

Ahmadi, J. and Arghami, N.R. (2003b). Comparing the Fisher Information in Record Values and iid Observations. Statistics, 37, 435-441.

Ahmadi, J. and Arghami, N.R. (2003a). Nonparametric Confidence and Tolerance Intervals from Record Values Data. Statistical Papers, 44, 455-468.

Ahmadi, J. and Balakrishnan, N. (2010). Prediction of Order Statistics and Record Values from Two Independent Sequences. Statistics, 44, 417-430.

Ahsanullah, M. (1995). Record Statistics. Commack, Nova Science, New York.

Arnold, B.C., Balakrishnan, N. and Nagaraja, H.N. (1998). Records. John Wiley and Sons, New York.

Asgharzadeh, A. and Fallah A. (2011). Estimation and Prediction for Exponentiated Family of Distributions Based on Records. Communications in Statistics-Theory and Methods, 40, 68-83.

Asgharzadeh, A., Valiollahi, R. and Raqab, M.Z. (2011). Stress-Strength Reliability of Weibull Distribution Based on Progressively Censored Samples. SORT, 35, 103-124.

Balakrishnan, N. and Aggarwala, R. (2000). Progressive Censoring: Theory Methods and Applications. Birkhäuser, Boston.

Balakrishnan, N., Ahsanullah, M. and Chan, P.S. (1995). On the Logistic Record Values and Associated Inference. Journal of Applied Statistical Science, 2, 233-248.

Balakrishnan, N. and Asgharzadeh, A. (2005). Inference for the Scaled Half-Logistic Distribution Based on Progressively Type II Censored Samples. Communications in Statistics-Theory and Methods, 34, 73-87.

Balakrishnan, N. and Kannan, N. (2000). Point and Interval Estimation for the Parameters of the Logistic Distribution Based on Progressively Type-2 Censored Samples. Handbook of Statistics, 20, 431-456.

Carlin, P.B. and Gelfand, A.E. (1993). ParametricLlikelihood Inference for Record Breaking Problems. Biometrika, 80, 507-515.

Chandler, K.N. (1952). The Distribution and Frequency of Record Values. Journal of Royal Statistical Society, B14, 220-228.

Chen, M.H. and Shao, Q.M. (1999). Monte Carlo Estimation of Bayesian Credible and HPD Intervals, Journal of Computational and Graphical Statistics, 8, 69-92. 
Efron, B. (1982). The Jackknife, the Bootstrap and Other Re-Sampling Plans. Philadelphia, PA: SIAM, vol. 38, CBMS-NSF Regional Conference Series in Applied Mathematics.

Ferguson, T.S. (1996). A Course in Large Sample Theory. Chapman and Hall, London.

Feuerverger, A. and Hall, P. (1998). On Statistical Inference Based on Record Values. Extremes, 12, 169-190.

Glick, N. (1978). Breaking Records and Breaking Boards. American Mathematical Monthly, $85,2-26$.

Gulati, S. and Padgett, W.G. (1995). Nonparametric Function Estimation from Inversely Sampled Record-Breaking Data. Canadian Journal of Statistics, 23, 359-368.

Gulati, S. and Padgett, W.J. (2003). Parametric and Nonparametric Inference from RecordBreaking Data. Lecture Notes in Statistics, 172, Springer-Verlag New York, Inc.

Hall, P. (1988). Theoretical Comparison of Bootstrap Confidence Intervals, Annals of Statistics, 16, 927-953.

MirMostafaee, S.M.T.K. and Ahmadi, J. (2011). Point Prediction of Future Order Statistics from an Exponential Distribution, Statistics and Probability Letters, 81, 360-370.

Nevzorov, V. (2001). Records: Mathematical Theory. In: Translation of Mathematical Monographs, vol. 194. Amer. Math. Soc. Providence, RI, USA.

Lee, H.M., Lee, W.C., Lei, C.L. and Wu, J.W. (2011). Computational Procedure of Assessing Lifetime Performance Index of Weibull Lifetime Products with the Upper Record Values. Mathematics and Computers in Simulation, 81, 1177-1189.

Raqab, M.Z., Asgharzadeh, A. and Valiollahi, R. (2010). Prediction for Pareto Distribution Based on Progressively Type-II Censored Samples. Computational Statistics and Data Analysis, 54, 1732-1743.

Robert, C.P. and Casella, G. (2004). Monte Carlo Statistical Method. Springer, New York.

Soliman, A.A. and Al-Aboud, F.M. (2008). Bayesian Inference Using Record Values from Rayleigh Model with Application. European Journal of Operational Research, 185, 659-672.

Tiku, M.L., Tan, W.Y. and Balakrishnan, N. (1986). Robust Inference. Marcel Dekker, New York. 


\section{Appendix}

Proof of (29). For $i \geqslant 1$, we have

$$
\begin{aligned}
E\left[X_{i}\left\{1-F\left(X_{i}\right)\right\}\right] & =\int_{-\infty}^{\infty} x\{1-F(x)\} \frac{[-\ln \{1-F(x)\}]^{i-1}}{(i-1) !} f(x) d x \\
& =\int_{0}^{1} F^{-1}(u)(1-u) \frac{\{-\ln (1-u)\}^{i-1}}{(i-1) !} d u \\
& =\int_{0}^{1}(1-u) \ln (u) \frac{\{-\ln (1-u)\}^{i-1}}{(i-1) !} d u \\
& +\int_{0}^{1}(1-u) \frac{\{-\ln (1-u)\}^{i}}{(i-1) !} d u,
\end{aligned}
$$

since $F^{-1}(u)=\ln u-\ln (1-u)$. Setting $t=-\ln (1-u)$, we get

$$
E\left[X_{i}\left\{1-F\left(X_{i}\right)\right\}\right]=\int_{0}^{\infty} \ln \left(1-e^{-t}\right) e^{-2 t} \frac{t^{i-1}}{(i-1) !} d t+\frac{i}{2^{i+1}}
$$

which, upon writing $\ln \left(1-e^{-t}\right)=-\sum_{l=1}^{\infty} \frac{e^{-l t}}{l}$, immediately gives

$$
E\left[X_{i}\left\{1-F\left(X_{i}\right)\right\}\right]=\frac{i}{2^{i+1}}-\sum_{l=1}^{\infty} \frac{1}{l(l+2)^{i}} .
$$

Proof of (30). We have

$$
\begin{aligned}
E\left[X_{i}^{2} F\left(X_{i}\right)\left\{1-F\left(X_{i}\right)\right\}\right]= & \int_{-\infty}^{\infty} x^{2} F(x)\{1-F(x)\} \\
& \times \frac{[-\ln \{1-F(x)\}]^{i-1}}{(i-1) !} f(x) d x \\
= & \int_{0}^{1}\{\ln u-\ln (1-u)\}^{2} u(1-u) \\
& \times \frac{\{-\ln (1-u)\}^{i-1}}{(i-1) !} d u .
\end{aligned}
$$


Using binomial expansion and setting $t=-\ln (1-u)$, we get

$$
\begin{aligned}
E\left[X_{i}^{2} F\left(X_{i}\right)\left\{1-F\left(X_{i}\right)\right\}\right]= & \int_{0}^{\infty} e^{-2 t}\left(1-e^{-t}\right)\left\{\ln \left(1-e^{-t}\right)\right\}^{2} \frac{t^{i-1}}{(i-1) !} d t \\
& +2 \int_{0}^{\infty} e^{-2 t}\left(1-e^{-t}\right)\left\{\ln \left(1-e^{-t}\right)\right\} \frac{t^{i}}{(i-1) !} d t \\
& +\int_{0}^{\infty} e^{-2 t}\left(1-e^{-t}\right) \frac{t^{i+1}}{(i-1) !} d t .
\end{aligned}
$$

Writing $\ln \left(1-e^{-t}\right)=-\sum_{l=1}^{\infty} \frac{e^{-l t}}{l}$ and using the expansion

$$
\left(\sum_{l=1}^{\infty} a_{l}\right)^{2}=\sum_{l=1}^{\infty} a_{l}^{2}+2 \sum_{1 \leqslant l<k<\infty} \sum_{l} a_{l} a_{k},
$$

we obtain

$$
\begin{aligned}
E\left[X_{i}^{2} F\left(X_{i}\right)\left\{1-F\left(X_{i}\right)\right\}\right]= & \sum_{l=1}^{\infty}\left\{\frac{1}{l^{2}(2 l+2)^{i}}-\frac{1}{l^{2}(2 l+3)^{i}}\right\} \\
& +2 \sum_{1 \leqslant l<k<\infty}\left\{\frac{1}{l k(l+k+2)^{i}}-\frac{1}{l k(l+k+3)^{i}}\right\} \\
& +2 i \sum_{l=1}^{\infty}\left\{\frac{1}{l(l+3)^{i+1}}-\frac{1}{l(l+2)^{i+1}}\right\} \\
& +i(i+1)\left[\frac{1}{2^{i+2}}-\frac{1}{3^{i+2}}\right] .
\end{aligned}
$$

\section{A. Asgharzadeh}

Department of Statistics,

University of Mazandaran,

Babolsar, Iran.

email: a.asgharzadeh@umz.ac.ir

\section{Abdi}

School of Mathematics and Computing, Higher Education Complex of Bam, Bam, Iran.

email:me.abdi.z@gmail.com

\section{R. Valiollahi}

Department of Statistics,

Semnan University,

Semnan, Iran.

email:valiollahi.reza@gmail.com 